Instituto Internacional de Investigación y Desarrollo Tecnológico Educativo INDTEC, C.A.

DOI: https://doi.org/10.29394/Scientific.issn.2542-2987.2021.6.21.20.376-395

OAI-PMH: http://www.indteca.com/ojs/index.php/Revista Scientific/oai

Ensayo Original / Original Essay

\title{
El Proceso de Enfermería: Horizonte Vocacional para el Cuidado
}

\author{
Autora: Yuli Toribia Rengifo de Vargas \\ Universidad Pedagógica Experimental Libertador, UPEL \\ yulireng@gmail.com \\ Puerto Cabello, Venezuela \\ https://orcid.org/0000-0002-1422-3745
}

\section{Resumen}

La vocación es la inspiración o sentimiento, que contempla una serie de cualidades que caracteriza a cada persona y orienta hacia una conducta o forma de vida, especialmente en su profesión. El cuidado es una función desempeñada por la enfermera, es su esencia, enfocada en apoyar el tratamiento a los pacientes a su cargo. En enfermería los profesionales que acceden a formarse como tal deben contar con esta virtud. El presente ensayo pretende en su objetivo general reflexionar acerca del proceso de enfermería: horizonte vocacional para el cuidado. En este ensayo, se hace una disertación sobre el desarrollo de la Enfermería, enmarcado en la vocación como herramienta fundamental para la aplicación del cuidado proporcionado por la enfermera, dejando a su vez una sensación de satisfacción en la relación terapéutica paciente-enfermera. Guiado por la Teoría del cuidado Humano de Watson (2008), como sustento teórico. Generando a su vez, efectos positivos, en la salud del paciente que el Proceso de Enfermería otorga, siendo este un método científico para la aplicación del cuidado a la persona, siendo la vocación casi un requisito para ser profesional enfermería, proporcionar cuidados es una tarea difícil que requiere ser realizada con amor y calidad, cuya finalidad es satisfacer las necesidades del paciente enfocados en la vocación visualizado como un horizonte para el cuidado.

Palabras clave: vocación; cuidado; esencia; enfermería. Código de clasificación internacional: 3204.03 - Salud profesional.

Cómo citar este ensayo:

Rengifo, Y. (2021). El Proceso de Enfermería: Horizonte Vocacional para el Cuidado. Revista Scientific, 6(21), 376-395, e-ISSN: 2542-2987. Recuperado de: https://doi.org/10.29394/Scientific.issn.2542-2987.2021.6.21.20.376-395

Fecha de Recepción: 09-02-2021
Fecha de Aceptación: 07-07-2021
Fecha de Publicación: 05-08-2021 


\title{
The Nursing Process: Vocational Horizon for Care
}

\begin{abstract}
The vocation is the inspiration or feeling, which contemplates a series of qualities that characterizes each person and guides them towards a behavior or way of life, especially in their profession. Care is a function performed by the nurse, it is the essence of her, focused on supporting the treatment of the patients in her charge. In nursing, the professionals who agree to be trained as such must have this virtue. The present essay aims in its general objective to reflect on the nursing process: vocational horizon for care. In this essay, a dissertation is made on the development of Nursing, framed in the vocation as a fundamental tool for the application of the care provided by the nurse, while leaving a feeling of satisfaction in the patient-nurse therapeutic relationship. Guided by the Theory of Human Care by Watson (2008), as theoretical support. Generating, in turn, positive effects on the health of the patient that the Nursing Process grants, this being a scientific method for the application of care to the person, being the vocation almost a requirement to be a nursing professional, providing care is a task difficult that requires to be carried out with love and quality, whose purpose is to satisfy the needs of the patient focused on the vocation visualized as a horizon for care.
\end{abstract}

Keywords: vocation; watch out; essence; nursing. International classification code: 3204.03 - Occupational health.

\section{How to cite this essay:}

Rengifo, Y. (2021). The Nursing Process: Vocational Horizon for Care. Revista Scientific, 6(21), 376-395, e-ISSN: 2542-2987. Recovered from: https://doi.org/10.29394/Scientific.issn.2542$\underline{2987.2021 .6 .21 .20 .376-395 ~}$

Date Received: 09-02-2021
Date Acceptance: 07-07-2021
Date Publication: 05-08-2021 


\section{Introducción}

El Proceso de Enfermería (PE), es un método científico que contempla cinco etapas secuenciales (valoración, diagnostico, planificación, ejecución y evaluación) para la aplicación del cuidado proporcionado por la enfermera al individuo, familia y comunidad. El abordar esta temática genera una perspectiva dirigida deliberar acerca del Proceso de Enfermería, la vocación y el cuidado, ya que la vocación repercute de manera positiva en la calidad y calidez del cuidado prestado al paciente.

El presente ensayo tiene como objetivo general reflexionar acerca del proceso de enfermería: horizonte vocacional para el cuidado. El Proceso de Enfermería es una herramienta que facilita a los profesionales de enfermería elementos para cuidar en búsqueda de la satisfacción de las necesidades o problemas del ser humano, al respecto el cuidado es la esencia del profesional de enfermería y éste se puede definir como actividades requeridas de valores personal y profesional, conducente a la promoción de salud, prevención de enfermedades y rehabilitación de la persona, a su vez fomenta la educación para la salud y el autocuidado. Esto involucra conocer al ser humano, mostrar interés por sus necesidades y afecciones, lo que demanda un esfuerzo de atención, una concentración a veces más agotadora que el esfuerzo físico mismo.

En este orden de ideas, la enfermera al aplicar cuidados apoyándose en el Proceso de Enfermería, considera elementos que van más allá del conocimiento adquirido, ya que, en su preocupación por cuidar del otro, mantiene un interés demostrado en sus acciones, que implican a su vez un gasto físico y de energía mental extra, al mostrar interés, preocupación, sentir solidaridad, entre otros aspectos dirigidos a dar calidad de vida.

Así mismo, Heidegger (1991), citado por Guevara, Zambrano y Evies (2001): "el filósofo por excelencia del cuidado, reconoce que éste es una constitución ontológica, siempre subyacente a todo lo que el ser humano 
emprende, proyecta y hace" (pág. 2). La realidad del profesional de enfermería se enmarca en el cuidado como su esencia, dada la naturaleza y percepción personal, desde una óptica vocacional, guarda relación con el ser, con el pensar y sentir para actuar de manera ideal.

Por su parte, Guerrero-Ramírez, Meneses-La Riva y De La Cruz-Ruiz (2016): aseguran que la "filosofía de Jean Watson sostiene que el Cuidado Humano comprende; un Compromiso Moral (Proteger y realzar la dignidad humana; va más allá de una evaluación médica), la experiencia, percepción y la conexión: (Mostrando interés profundo a la persona)" (pág. 137). Es decir que el cuidado humano contempla, atender al paciente desde una visión holística, con un amplio compromiso que guarda una estrecha relación con la vocación de la enfermera, que formula a su vez, aquello propio del ser humano que les motiva a vivir de una manera determinada, en relación con un oficio o profesión, que se desarrolla y se hace parte de la historia o proyecto de vida del ser.

El hombre en su vocación profesional, encuentra un horizonte para realizarse como persona, en donde el conocimiento es indispensable para la formación profesional, permitiéndose desempeñarse en el campo laboral, en donde la enfermera, al desarrollar el Proceso de Enfermería, logra satisfacer las necesidades del individuo, familia y comunidad al dar cuidados de calidad. En ocasiones la vocación viene innata en la persona, y es donde desde pequeño, muestra una inclinación profesional o deseo de hacer una carrera, pero de no ser así, algunos se guían por aquello que le gusta hacer y le aplica una cuota de valor personal.

En enfermería el marco para el cuidado se inicia desde la valoración, que es indispensable para la supervivencia. El cuidado requiere habilidades practicas relacionadas con la conducta propias y el pensamiento con criterio científico (conocimiento). El cuidado se considera la esencia la esencia de enfermería dimensionada en la terapéutica profesional., que además es un 
valor fundamental en praxis de enfermería, dirigiría a satisfacer las necesidades del individuo, familia y comunidad.

En este contexto la competencia de enfermería desde la perspectiva de liderazgo, como compromiso moral, requieren contar con un método para obtener de información del paciente, esto se logra a través del Proceso de enfermería, que implica creatividad, conciencia y conocimientos actualizados para satisfacer las necesidades y o cuidados del ser al que se le está proporcionando cuidados.

Es importante considerar por otra parte, aquellos aspectos referentes al cuidado, los mismos concerniente a las características personales de la enfermera(o), ya que se debe considerar como un problemas importante el hecho de que el paciente debe ser tratado en todo momento como un ser humano y no como una patología, número de cama o cuarto, es decir que la persona cuidada es un sujeto no un objeto, por lo tanto merece respeto y ser tratado como ser humano en todo momento, dirigirse a él con su respectivo nombre o apellido y no hacerlo por su patología o número de cama.

En este orden de ideas, es de considerarse que el paciente no debe estar limitado a verse como un elemento en beneficio de otros. Todo paciente requiere ser cuidado con la finalidad de preservar la existencia, esta espera que su condición de salud mejore o mejor aún, que su problema sea eliminado, y por tanto el respetar su vida y su integridad, debe ser prioridad sin ser expuesto a riesgos.

En base a las consideraciones anteriores, Hernández (2018a): refiere en su ensayo que "[...] a medida que los profesionales de enfermería desempeñen sus funciones en forma óptima, la sociedad se beneficiará de una atención basada en modelos de calidad y excelencia propios de las políticas de estado [...]" (pág. 351). Es de gran relevancia lo establecido dentro de la Constitución de la República Bolivariana de Venezuela (CRBV, 1999): referente a los derechos a la salud de los venezolanos dentro del país, estas 
políticas de salud contemplan estrategias en donde se cuenta con tres niveles de salud. Nivel I: Promoción de salud; Nivel II: Prevención de enfermedades; y Nivel III: Rehabilitación, que van desde la promoción, prevención y rehabilitación del paciente.

Todo esto refleja el alcance y beneficio del cuidado de enfermería, centrado en la competencia y valores humanos, para mantener la salud y preservar la vida de las personas sanas y enfermas, contando con un soporte legal contemplado en la Ley de Ejercicio de Enfermería de la República Bolivariana de Venezuela, lo que permite abordar la temática con una perspectiva dirigida deliberar acerca del Proceso de Enfermería desde la vocación para aplicar el cuidado, temiendo presente que la vocación repercute de manera positiva en la calidad y calidez del cuidado prestado al paciente. De allí que el objetivo general del ensayo es reflexionar acerca del Proceso de Enfermería en un horizonte vocacional para el cuidado.

\section{Desarrollo}

\subsection{Horizonte vocacional para el cuidado}

Fundamentalmente la historia de enfermería se edifica a partir de los cuidados que son proporcionados para garantizar la continuidad de la vida de la persona, de allí que el cuidado es considerado por muchos autores desde tiempos muy remotos y hasta ahora, como la esencia de la enfermera. En su preocupación por asegurar la continuidad de la vida, la planificación y ejecución de las actividades de enfermería para el cuidado del paciente, es realizada de manera holística, a través del Proceso Enfermero en el desarrollo de sus cinco etapas: 1. Valoración, 2. Diagnóstico, 3. Planificación, 4. Ejecución, y 5. Evaluación del cuidado. Es una herramienta que apoya al personal de enfermería para lograr la supervivencia. Reseñado en la historia, el hombre se dedica a la caza para alimentarse, a manejar armas para defenderse, a recolectar plantas para aprender como reproducirlas, a observar 


\section{Ensayo Original / Original Essay}

la naturaleza para entender su entorno, etc.

Hablar de vocación encierra un sentimiento o llamado interno que vislumbra lo que se desea ser y hacer en relación con una profesión u oficio. Para enfermería la vocación se considera un factor primordial de considerar, al decidir formarse para cuidar a otros. Esta es reflejo de la personalidad de la profesionalidad de cada enfermera, en el ejercicio de sus funciones.

Según Nava (2012): "la dedicación y el amor hacia nuestra profesión los ejerce cada uno; ésta es la visión que debe tener cada profesionista en su práctica diaria y cumplirla con la más alta calidad" (pág. 62). Es decir que la vocación viene inmersa en los valores y principios que enmarcan las cualidades de los enfermeros, es un reflejo del cuidado demostrado por el profesional de enfermería en su práctica.

La disposición es casi un requisito indispensable para ser profesional enfermería, ya que proporcionar a otros cuidados es una tarea difícil, se necesita vocación de servicio para ello, a pesar de contar con el Proceso de Enfermería, que es una herramienta o instrumento con apoyo científico, desarrollado actualmente para la satisfacción de las necesidades y/o problemas de las personas de manera individual.

Decir vocación es referirse al llamado interno que la persona percibe como propósito de vida, dirigido a realizar labores en donde ontológicamente hay una interacción entre el ser que cuidada y el ser cuidado, enmarcado en la vocación, como un valor humano de la enfermera, tener disposición propia, radicada en la idea del ser humano y complementada con los más altos estándares de formación, para asumir el ejercicio profesional.

Es relevante, lo referido por Rivera y Herrera (2006): "cuidar comprensivamente es percibir intencionadamente ese mundo privado dinámico, que se va develando en la medida que se profundiza en la relación de cuidado" (pág. 162). Es decir que el cuidar es más que acompañar, es una estructura que se va construyendo en el proceso de relación enfermera- 
paciente, en donde ambos deben aprender a establecer puentes de comunicación, indispensable para comprender el significado la vocación para cuidar. Con relación a esto, Hernández (2018b), refiere que:

[...] La formación del profesional de enfermería lleva consigo una serie de elementos axiológicos, teóricos y humanistas que construyen los saberes enfermeros y permiten la reflexión crítica constante del cuidado brindado, aflorando así la vocación como factor indispensable en la esencia de esa acción humana [...] (pág. 352).

Por todo esto, es importante resaltar que la vocación forja sus cimientos en esa voz interior, que orienta a la persona a realizarse en función de su proyecto de vida. La vocación de enfermería está estrechamente sujeta al ejercicio profesional, enfocado en esos valores que conllevan a una internalización del proceder y el actuar con conciencia, conocimiento y sentido de pertenencia, fundamental como razón de ser del cuidado humano en el Proceso de Enfermería.

\subsection{El proceso de enfermería}

Uno de los grandes obstáculos para enfermería, en épocas pasadas, era la falta de un componente científico de conocimiento especial, que ayudara a la solución de los problemas del paciente. Se destaca el apoyo y beneficio otorgado por el Proceso de Atención de Enfermería o Proceso de Enfermería como ciencia del cuidado, en donde se pone en práctica las habilidades y destrezas del profesional de enfermería para satisfacer las necesidades del usuario, familia y comunidad.

El proceso de Enfermería está presente en cada una de las acciones de la enfermera, para aplicar el cuidado a las personas, ya sea que estén sanas o enfermas. Al respecto, la Organización Panamericana de la Salud y la Organización Mundial de la Salud (OPS-OMS, 2018), en donde el Director 
Instituto Internacional de Investigación y Desarrollo Tecnológico Educativo INDTEC, C.A.

DOI: https://doi.org/10.29394/Scientific.issn.2542-2987.2021.6.21.20.376-395

OAI-PMH: http://www.indteca.com/ojs/index.php/Revista Scientific/oai

Ensayo Original / Original Essay

del Departamento de Sistemas y Servicios de Salud, James Fitzgerald, destaca que:

La enfermería puede desempeñar una función crucial en el avance de la APS. Los nuevos perfiles como el personal de enfermería de práctica avanzada [...] pueden ser fundamentales en este esfuerzo, y en particular, en la promoción de la salud, la prevención de enfermedades y la atención, especialmente en zonas rurales y subatendidas (pág. 10).

Significa esto, que al proveer de autonomía a los profesionales de enfermería, se les incrementa su responsabilidad y por ende, su capacidad de intervención, en los cuidados de la salud, tanto en espacios citadinos, como en zonas rurales, para brindar Atención Primaria de Salud (APS), en la persona individualmente, en las familias y en las comunidades. Dando así mayor alcance con las actividades realizadas por profesionales como el de las Enfermeras de Práctica Avanzada (EPA), en la promoción de salud, prevención de enfermedades y disminución de la morbi-mortalidad en la población.

Refieren Vele y Veletanga (2015): que actualmente, "el Proceso de Atención de Enfermería (P.A.E) es considerado como un método científico que posibilita al enfermero/a realizar planes de cuidados en forma directa e individualizada, de manera racional, lógica y sistemática [...] (pág. 16). Este proceso es calificado como la base del ejercicio de la profesión, sustentado científicamente por la aplicación del método científico, en la práctica asistencial, en donde radica su importancia.

Es una herramienta profesional aplicable e indispensable para planear el cuidado al individuo, familia y comunidad, permitiendo un vínculo entre el paciente y la enfermera, teniendo esta una cualidad reconocida como vocación de servicio, que le permite cuidar y sentir amor por su trabajo.

Además de ser un instrumento que se implica en la profesión, también 
lo hace sobre la relación terapéutica enfermera-paciente, que permite generar un ambiente armónico, de confianza y seguridad para ambos en el desarrollo del proceso de enfermería, para la aplicación del cuidado humano. Es importante mencionar que la enfermería contempla en sus áreas de desempeño laboral cinco actividades a cargo: Asistencial (atención directa al paciente), Administrativa (actividades gerenciales), Docente (educación continua y formación profesional), Gremial (actividades del gremio) e Investigadora (estudios de investigación científica). Esta última se realiza través de este instrumento, en donde la enfermera puede realizar su actividad docente e investigadora, en busca de satisfacer aquellos problemas 0 necesidades del paciente que les son esencial, aplicable igualmente en la familia y la comunidad.

Para el Consejo internacional de enfermeras (CIE, 2006): "la enfermera será personalmente responsable y deberá rendir cuentas de la práctica de enfermería y del mantenimiento de su competencia mediante la formación continua" (pág. 3). Ante esto, queda establecida formalmente la responsabilidad que tiene el profesional de enfermería para velar por la calidad de los cuidados proporcionados al paciente.

Por lo tanto, se expresa la calidad de cuidados y es puesta en evidencia al momento de prestar el servicio al individuo, manejando un juicio crítico, enmarcado en el conocimiento para satisfacer sus necesidades y brindarles seguridad en su entorno. La seguridad de los pacientes es definida por la Organización Mundial de la Salud (OMS, 2009), citado por Villarroel y Febré (2016), como:

La ausencia de riesgo o daño potencial asociado con la atención sanitaria, que se basa en el conjunto de elementos estructurales, procesos, instrumentos y metodologías basadas en evidencias científicamente probadas con el objeto de minimizar el riesgo de sufrir un evento adverso en el proceso de atención de salud o de mitigar sus consecuencias (pág. 92). 
En consideración a lo antes expuesto, los riesgos se presentan en cualquier contexto, y la atención a la salud no escapa a estas inseguridades, por tal razón es necesario disminuir los factores predisponentes a estos eventos, que pudieran ocurrir en el marco de la seguridad del paciente, en donde el personal de salud, especialmente las enfermeras, juegan un papel importante en atención a la salud de los individuos, la familia y la comunidad, en la promoción, prevención y rehabilitación en torno al bienestar.

Con relación a los cuidados actuales, para Duarte, Gómez y Sánchez (2007): la cultura para brindar estos, se encuentra presente en las opiniones de la enfermera, a través de los valores que forman parte del perfil de la enfermera, contemplando el conocimiento requerido para la praxis profesional.

El Proceso de Enfermería contempla en su estructura, cinco etapas a desarrollar, para el cuidado humano al individuo, familia y comunidad, estas son:

1. Valoración: se refiere a la recogida de datos del paciente. Datos subjetivos (anamnesis) y datos objetivos (examen físico).

2. Diagnóstico: es el análisis e interpretación de los datos recopilados en la valoración.

3. Planificación: considera establecer acciones a desarrollar para resolver el problema captado. Estrategias.

4. Ejecución: corresponde a la aplicación del cuidado (intervención y actividades).

5. Evaluación: son los resultados obtenidos.

\subsection{Etapas del proceso de enfermería}

El Proceso de enfermería es una herramienta que permite planificar los cuidados al paciente, a través de cinco tapas sistemáticas que permiten realizar la valoración al paciente, obteniendo datos subjetivos (lo referido por el paciente o la familia) y datos objetivos (lo percibido por el enfermo). 
seguidamente estos datos son analizados para determinar el diagnostico de enfermería que puede ser: real de riesgo o potencia colaborativo.

Seguimos con la planificación: esta etapa permitirá diseñar un plan de cuidados en búsqueda de la solución a los problemas o necesidades encontradas, que serán ejecutadas. La ejecución es la cuarta etapa en donde se pondrá en práctica los cuidados planificados y organizados, finalmente la quinta etapa es la evaluación; allí obtendremos resultados de los cuidados planificados.

Figura 1. Proceso de enfermería.

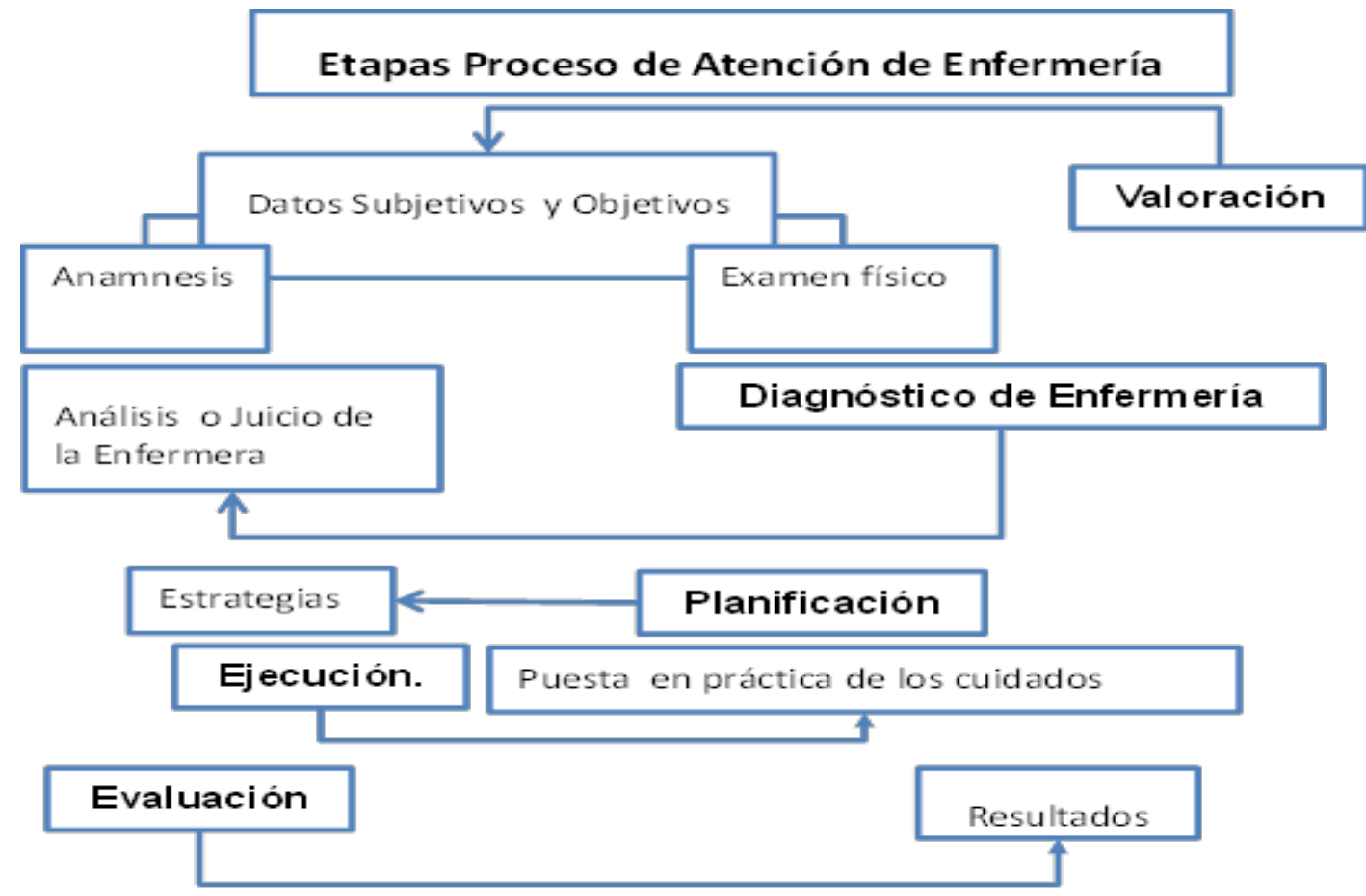

Fuente: Berman, Snyder, Kozier y Erb (2008); adaptación de la Autora (2020).

Se expresa en la figura 1, de manera sintetizada el Proceso de enfermería, sus etapas y elementos, el cual puede ser interpretado y analizado para la comprensión de esta, desarrollándola con habilidad y destreza en el campo laboral. 
Enmarcado en este proceso cabe resaltar que existe para sustento de este método y a la hora de aplicar el cuidado, los modelos, teorías y filosofía en enfermería, entre ellas la filosofía del cuidado humano, permitiendo que los profesionales de enfermería desarrollen el Proceso de Enfermería en sus cinco etapas, de manera sistemática, racional y con juicio crítico.

\subsection{El Ser humano y el Cuidado humano de enfermería}

De acuerdo con Alligood y Tomey (2011): "Virginia Henderson consideraba al paciente como un individuo que precisaba ayuda para conseguir independencia e integridad o integración total de mente y cuerpo" (pág. 55). El ser humano es percibido por la autora como una persona que tiene incorporado una serie de necesidades y por lo tanto requiere de apoyo o ayuda por parte del profesional de enfermería.

El ser humano requiere desarrollar actividades laborales que les son inseparable a sus capacidades, esto generalmente le es emocionalmente satisfactorio, y más aún, cuando lleva una carga de motivación o vocación. En enfermería, los profesionales son humanos formados para cuidar a humanos.

El profesional de enfermería en su naturaleza social y bajo una óptica humanista, está centrada en ayudar a otras personas, solicitantes del cuidado a reforzar potencialidades y/o minimizar desequilibrios en su salud, reconociendo que el ser humano es complejo, misterioso, formado por diversas dimensiones como persona humana

En torno a esto, la enfermera debe contar con una naturaleza humanista innata, que le permita facilitar la ayuda a prestar al individuo que solicita sus cuidados, con la finalidad de satisfacer aquellos requerimientos en torno al desequilibrio de la salud de la persona. De allí que debe mostrarse al ser como estructura conformada por cuerpo, mente y espíritu, es un ser complejo dimensionado. 
El cuidado se considera como un cúmulo de actos de vida, en donde la su finalidad o función es preservar la vida de los seres vivos para perpetuarse. En cuanto al cuidado con frecuencia, es el acto de relacionarse los seres humanos con otros, para ayudarle con la intención de resguardar la vida humana.

El complemento del cuidado es el afecto y tiene inmerso un valor humano, considerado como una necesidad. Por otra parte, Maslow (1991): en su pirámide de las necesidades básicas hace una jerarquización de ellas, en donde la necesidad de afecto forma parte de estas, ya que permite una interconexión entre las personas, el entorno e incluso con los objetos. La enfermera en su rol de cuidadora, establece para su relación terapéutica el afecto, constituido por Escobar (2004a), como el:

Involucramiento emocional o sentimiento de empatía por la experiencia del paciente. Sentimiento de preocupación o interés, vigilar el cuidado con protección, sentimiento de dedicación... respuesta que focaliza un incremento de la intimidad enfermerapaciente que favorece la auto actualización mutua y comprende cuatro etapas de desarrollo: la vinculación, constancia, intimidad y la confirmación; cada una con sus tareas propias. Si las etapas no se cumplen todas, el cuidado no tiene lugar (pág. 56).

Ante esto el afecto para enfermería, representa un elemento unido al cuidado humano, y es esencial para desarrollar el Proceso de Enfermería. Por tanto, la comunicación es básica en el desempeño laboral de la enfermera, para que ese proceso de comunicación sea efectivo, se requiere de una relación terapéutica sostenida en el afecto demostrado durante la aplicación del cuidado humano. El cuidar como afecto, genera un ambiente armónico, bidireccional para dar y recibir cuidado.

Adicionalmente, Escobar (2004b): lo enmarca en la constancia de las acciones, la paciencia que ofrece tolerancia, intimidad que orienta a la confianza y al respeto por el otro y la confirmación a través de la reevaluación 


\section{Ensayo Original / Original Essay}

del Proceso Enfermera(o). El afecto es una necesidad sentida, requerida y apreciada tanto por el cuidador como la persona cuidada en la relación enfermera-paciente.

En la práctica de enfermería, el acto de cuidar implica actuar centrado en valores, teñidos por la vocación profesional, para tal fin es fundamental los conocimientos que esta adquiere durante su formación académica y que va perfeccionando con la práctica diaria. Aunado a esto y desde una perspectiva innovadora que acopla la ciencia y el arte de enfermería centrando su atención en la persona, que interactúa con su entorno para adaptase a este, en todas sus vivencias y experiencias de salud.

El cuidado de enfermería se sustenta científicamente con la teoría de Cuidado Humano de Watson (2008a): la cual representa un compromiso profesional, motivador y esencial en el proceso de cuidado; que contempla un sistema centrado en normas éticas, basado en un enfoque humanista. El cuidado humano, enmarca su teoría en la energía del ser y la caridad de la persona que proporciona cuidado, teniendo como ejes los valores morales.

Al Valorar, identificar y evaluar el cuidado humanizado que brindan los profesionales de enfermería a los pacientes, se permite fortalecer el cuidado en las instituciones asistenciales; para restaurar el cuidado y la sanación como un arte. que constituye la base de la acción de enfermería. De igual manera, Watson (2008b): reconoce que es la esencia fundamental del ser, el querer cuidar, se pudiera considerar un acto primitivo o instintivo, en donde los seres humanos se relacionan de manera afectiva entre sí.

El cuidado humano de Watson (2008c): contiene premisas básicas que reflejan elementos interpersonales, transpersonales, espirituales por los que pasa el ser. Revela a su vez aquellas opiniones referentes a la cultura y creencias de la persona dentro del contexto de salud, que se enmarcan en el conocimiento para la aplicación del cuidado humano. 
Ensayo Original / Original Essay

\section{Conclusiones}

Ser enfermera significa cuidar y ese cuidado, amerita contar con profesionales con cualidades generadas del ser. Para cuidar con calidad al paciente se necesita conocimiento, que es indispensable, y adquirido en la formación académica, este es fortalecido en la práctica. Pero más allá de ese elemento cognitivo, está ese llamado interno de la enfermera, conocido como vocación, que guía a la persona a elegir la profesión que desea realizar como complemento de vida. Esto implica conocer al ser humano, mostrar interés por sus necesidades y afecciones, lo que demanda una carga emocional y esfuerzo de atención, una concentración a veces más agotadora que el esfuerzo físico mismo.

Cuidar es la búsqueda de la satisfacción de las necesidades o problemas del ser humano, teniendo presente que el cuidado es la esencia del profesional de enfermería, la naturaleza misma que caracteriza su profesionalidad. En complemento y apoyo para el cuidado humano, enfermería como ciencia, cuenta con el Proceso de Enfermería, una herramienta que facilita a estos profesionales de la salud elementos para cuidar, en búsqueda de resultados óptimos que lleven a la satisfacción de las necesidades del ser.

Es significativo resaltar que la vocación tiene una estrecha relación con la disposición de ayuda de la enfermera para prestar cuidado humano, es por sí mismo, un horizonte en el que se visualiza la persona, como proyecto de vida.

Reseñando la teoría del Cuidado Humano, puedo decir que se enmarca un compromiso del profesional de enfermería, representado en la esencia que es ese cuidado propio de la enfermera para desarrollar el Proceso de Enfermería. De igual manera, esto comprende un sistema centrado en los valores y la ética, dentro de un contexto humanista y holístico.

Es concluyente, ante todo esto que la naturaleza de enfermería, está fundamentada no solo en el conocimiento, habilidades y destrezas, sino 
Ensayo Original / Original Essay

también en las motivaciones y afectos demostrados en su práctica profesional, al aplicar el cuidado humano a través del Proceso de Enfermería, la guía vocacional de un profesional es un soporte para cristalizar su proyecto de vida, en donde la persona se sienta satisfecho con sigo mismo y con los demás.

\section{Referencias}

Alligood, M., \& Tomey, A. (2011). Modelos y teorías en enfermería. Séptima edición. ISBN: 978-84-8086-716-0. Barcelona, España: Elsevier España, S.L.

Berman, A., Snyder, S., Kozier, B. \& Erb, G. (2008). Fundamentos de Enfermería. Volumen 1, ISBN: 978-84-8322-836-4. Madrid, España: Pearson Educación, S.A.

CIE (2006). Código deontológico del CIE para la profesión de enfermería. ISBN: 92-95040-43-0. Ginebra, Suiza: Consejo Internacional de Enfermeras.

CRBV (1999). Constitución de la República Bolivariana de Venezuela. Gaceta Oficial Extraordinaria N. ${ }^{\circ} 36.860,30$ de diciembre. Caracas, Venezuela: Asamblea Nacional Constituyente.

Duarte, G., Gómez, J., \& Sánchez, M. (coord.). (2007). Fundamentos teóricos de la enfermería comunitaria. ISBN: 978-84-96690-43-1. España: Enfo Ediciones.

Escobar, D. (2004a,b). Perspectiva Fenomenológica sobre el Poder del Cuidado Humano Amoroso. 1ra. Edición, Depósito Legal: IF04120048003284. Valencia, Venezuela: Instituto Vocacional de Venezuela - INSTIVOC.

Guevara, B., Zambrano, A., Evies, A. (2001). Cosmovisión en el cuidar de sí y cuidar del otro. Enfermería Global, 10(21), 1-7, e-ISSN: 1695-6141.

Recuperado de: https://revistas.um.es/eglobal/article/view/115281

Guerrero-Ramírez, R., Meneses-La Riva, M., \& De La Cruz-Ruiz, M. (2016). 
Cuidado humanizado de enfermería según la teoría de Jean Watson, servicio de medicina del Hospital Daniel Alcides Carrión. Lima - Callao, 2015. Revista Enfermería Herediana, 9(2), 133-142, eISSN: 2075-4000. Recuperado de:

https://doi.org/10.20453/renh.v9i2.3017

Hernández, R. (2018a,b). La Praxis de Enfermería: Una Vocación con Sentido Axiológico y Humanista. Revista Scientific, 3(9), 348-361, eISSN: 2542-2987. Recuperado de:

https://doi.org/10.29394/Scientific.issn.2542-2987.2018.3.9.19.348-361 Kérouac, S., Pepin, J., Ducharme, F., Duquette, A., \& Major, F. (1996). EI pensamiento enfermero. Primera edición, ISBN: 2-7607-0584-6; ISBN: 978-84-458-0365-3. Barcelona, España: Elsevier Doyma, S.L.

Maslow, A. (1991). Motivación y Personalidad. ISBN: 978-84-87189-84-5. Madrid, España: Ediciones Díaz de Santos, S.A.

Nava, M. (2012). Profesionalización, vocación y ética de enfermería. Revista de Enfermería Neurológica, 11(2), 62, e-ISSN: 1870-6592. Recuperado de:

https://www.medigraphic.com/cgibin/new/resumen.cgi?IDARTICULO $=38112$

OPS-OMS (2018). Ampliación del rol de las enfermeras y enfermeros en la atención primaria de salud. ISBN: 978-92-75-32003-7. Washington, D.C., Estados Unidos: Organización Panamericana de la Salud. Recuperado de: https://doi.org/10.37774/9789275320037

Rivera, M., \& Herrera, L. (2006). Fundamentos fenomenológicos para un cuidado comprensivo de enfermería. Texto \& Contexto Enfermagem, 15, 158-163. Recuperado de:

https://www.redalyc.org/articulo.oa?id $=71414366019$

Vele, S., \& Veletanga, D. (2015). Aplicación del proceso de atención de enfermería de las enfermeras/os, que laboran en el hospital 
Instituto Internacional de Investigación y Desarrollo Tecnológico Educativo INDTEC, C.A.

DOI: https://doi.org/10.29394/Scientific.issn.2542-2987.2021.6.21.20.376-395

OAI-PMH: http://www.indteca.com/ojs/index.php/Revista Scientific/oai

Ensayo Original / Original Essay

regional Vicente Corral Moscoso, Cuenca 2015. Tesis de Pregrado.

Cuenca, Ecuador: Universidad de Cuenca. Recuperado de:

https://dspace.ucuenca.edu.ec/handle/123456789/23234

Villarroel, X., \& Febré, N. (2016). Características de documentos utilizados

para estructurar las políticas internas relacionadas a las metas

internacionales de seguridad de pacientes. Ciencia y Enfermería,

22(2), 91-102, e-ISSN: 0717-2079. Recuperado de:

http://dx.doi.org/10.4067/S0717-95532016000200007

Watson, J. (2008a,b,c). Nursing: Human Science and Human Care. 1 Revised edition, ISBN: 978-0763753221. United States: Jones \& Bartlett Learning. 


\section{Yuli Toribia Rengifo de Vargas}

e-mail: yulireng@gmail.com

Nacida en el estado Yaracuy, Venezuela, el 5 de junio del

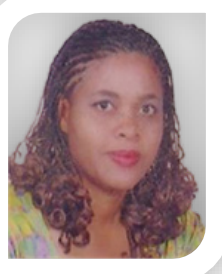
año 1965. Licenciada en Enfermería por la Universidad de Carabobo (UC); Magister en Enfermería Gerontológica y Geriátrica por la Universidad de Carabobo; Magister en Gerencia en Salud Pública por la Caribbean International University (CIU); docente del Instituto Universitario de Tecnología Juan Pablo Pérez Alfonso (IUTEPAL), Puerto Cabello (20022018); docente de la Universidad Nacional Experimental Politécnica de la Fuerza Armada (UNEFA, 2005), Puerto Cabello; docente del PNF de Enfermería de la Universidad Nacional Experimental Francisco de Miranda (UNEFM, 2012), Municipio Silva, Falcón, Venezuela.

El contenido de este manuscrito se difunde bajo una Licencia de Creative Commons ReconocimientoNoComercial-Compartirlgual 4.0 Internacional 